\title{
Topical fluorides and decalcification around fixed orthodontic appliances
}

\author{
Which topical fluoride preparations are best able to prevent decalcification \\ around fixed orthodontic appliances?
}

\author{
Chadwick BL, Roy J, Knox J, Treasure ET. The effect of topical \\ fluorides on decalcification in patients with fixed orthodontic \\ appliances: a systematic review. Am J Orthod Dentofacial Orthop \\ 2005; 128:601-606
}

Data sources Medline, Embase, Cochrane Library databases and the York Centre for Reviews and Dissemination Database of Abstracts of Reviews of Effectiveness were searched back to their inception. To identify recent nonindexed literature, a search by hand was also made of abstracts of articles published in proceedings of international, British, Australian, New Zealand, continental European, Japanese, Scandinavian and South American divisions of the International Association for Dental Research and of journals publishing orthodontic and fluoride research. Manufacturers of fluoride preparations were contacted to identify relevant unpublished data.

Study selection Studies were included if they were conducted on human subjects (not extracted teeth) undergoing orthodontic treatment with fixed appliances; if the study design included comparison groups (randomised controlled trials, clinical trials, and prospective observational studies with concurrent or historical comparison groups); if they used topical fluoride; and if they had a valid, clearly described and reproducible outcome measure of decalcification both before and after orthodontic treatment (ie, severity of white spot lesions, decayed or missing teeth and teeth with filled surfaces). Nonhuman, laboratory-based studies not using fixed appliances or topical fluorides were excluded.

Data extraction and synthesis Studies were considered against predetermined criteria. Initially, titles and abstracts were evaluated by two reviewers, and if there was any doubt the article was retrieved. All excluded titles and abstracts were rechecked by a third examiner. All studies that met the criteria were read by two reviewers to ensure that defined outcome measures were used. Those not meeting the appropriate outcome-measure requirement or lacking a comparison group were excluded. Included studies were double-extracted onto data extraction sheets. Disagreements were resolved by the third team member. Effect modifiers were identified, eg, level of water fluori dation, caries status, oral hygiene and age, and recorded if reported. From the data presented in the studies it was not possible to calculate the confidence intervals of the mean difference between groups so the results were described qualitatively. A preventive fraction (PF), ie, the difference in caries increments between treatment and control groups expressed as a proportion of the increment in the control group, was calculated. Note that these must be viewed with extreme caution because they have no confidence intervals.

Results Searches identifi ed 503 titles and abstracts: 143 relevant articles were retrieved of which 45 were excluded because of study design, 76 because they did not meet the inclusion criteria, and nine because they did not contain data for decalcification at baseline. This left 13 articles of which only six could be included because of missing data. As these studies used different methodologies and reporting strategies, it was not possible to undertake a meta-analysis, so a qualitative analysis was carried out.

Address for correspondence: Dr BL Chadwick, Dental School, Cardiff University, Cardiff, Wales CF14 4XY, UK. E-mail, chadwickbl@cardiff.ac.uk.
Conclusions The use of topical fluorides in addition to fluoride toothpaste reduced the incidence of decalcification in populations with both fluoridated and nonfluoridated water supplies. Different preparations and formats appear to decrease decalcification, but there was no evidence that any one method was superior. There was some evidence that the potency of fluoride preparations might be important.

\section{Commentary}

Fluorides are much-documented in the dental literature, where the efficacy of various topical fluorides has already been the subject of several systematic reviews. ${ }^{1-7}$ It is interesting, however, to examine a review that focuses on the specific issue of topical fluorides and their relation to enamel decalcification in patients who are undergoing fixed orthodontic treatment.

Dental caries, in spite of a considerable body of research, continues to be enigmatic and challenging. An inherent susceptibility to dental caries could be compounded by any additional plaque buildup around an orthodontic appliance, a subsequent alteration of oral microflora and an increase in the mutans streptococci implicated in enamel decalcification. So what should a concerned clinician do or prescribe? If you choose to reinforce oral hygiene measures, which is the most appropriate vehicle of delivery? The systematic review has a well-defined objective and clinical bottom-line. It grabs the attention immediately in defining the clinical imperatives for an orthodontist.

The review is well thought out and is carefully structured, with much attention to detail. The search strategy is comprehensive, covering a complete spectrum of print, electronic media and personal communications with authors. The inclusion and exclusion criteria are well-defined, with data validation and abstraction based on a threestage process with two reviewers assessing the studies at any point in the process. The definition of objective outcome measures of decalcification both before and after orthodontic treatment strengthens the database selection. A filtered and multilayered scrutiny, both for included and excluded studies, does yield the best evidence. It is not surprising that only six studies find their place in th e review out of a possible 143 . Once again, a review highlights the paucity of good evidence in orthodontics.

One of the strong features of this review is the effort to deal specifically with effect-modifiers. After all, enamel decalcification is a multifactorial entity and all contributory factors need to be specifically taken into account.

The one area of difficulty in the review in terms of data abstraction and presentation is the difference in presentation of results, with different groups using various outcome measures. This does raise an issue on the comparability of studies and the results. The choice of PF to represent the proportion of caries reduction in the absence of confidence intervals is rational. This would allow some flexibility and standardisation of outcomes. As stated by the authors, nonconformity of reporting data has been noted in other systematic reviews.

The review makes it possible to identify trends and draw conclusions, which the authors have done. The trends are clear: as well as using fluoride toothpaste, the addition of a topical fluoride will be 
effective in reducing enamel decalcification in patients who have fixed orthodontic appliances. The effect will be universal, occurring in both individuals who have fluoridated and nonfluoridated water supplies.

The strength of the topical fluoride is of significance. The review does not, however, conclusively establish the best vehicle for delivery, so the choice of a gel, rinse, varnish or mouthwash is best left to the clinician and/ or patient. The review also cannot support or identify which preparation or schedule would be most effective. Therein lies the scope for further well-structured studies: the last word on caries prevention is yet to be written. There is also an issue over the deleterious effect of high-potency fluorides on the orthodontic hardware and the oral environment, probably also an area for future study. The bottom line is clear, nevertheless: all orthodontic patients who have fixed appliances should use fluoride toothpastes, and the addition of any topical fluoride would further reduce the risk of enamel decalcification. This is an excellent review.

\section{Practice point}

- The regular use of fluoride toothpaste should be reinforced during fixed appliance therapy and the use of additional topical fluoride encouraged.

\section{Anmol S Kalha}

Department Of Orthodontics, Centre for Evidence Based Dentistry, College of Dental Sciences, Davangere, India
1. Petersson LG, Twetman S, Dahlgren H, Norlund A, Holm AK, Nordenram G,et al. Professional fluoride varnish treatment for caries control: a systematic review of clinical trials. Acta Odontol Scand 2004; 62:170-176.

2. Benson PE, Parkin N, Millett DT, Dyer FE, Vine S, Shah A. Fluorides for the prevention of white spots on teeth during fixed brace treatment. Cochrane Database Syst Rev 2004; Issue 3. Art. No.: CD003809

3. Marinho VC, Higgins JP, Sheiham A, Logan S. Combinations of topical fluoride (toothpastes, mouthrinses, gels, varnishes) versus single topical fluoride for preventing dental caries in children and adolescents. Cochrane Database Syst Rev 2004 2004, Issue 1. Art. No.: CD002781

4. Marinho VC, Higgins JP, Sheiham A, Logan S. One topical fluoride (toothpastes, or mouthrinses, or gels, or varnishes) versus another for preventing dental caries in children and adolescents. Cochrane Database Syst Rev 2004; Issue 1. Art. No.: CD002780

5. Marinho VC, Higgins JP, Logan S, Sheiham A. Topical fluoride (toothpastes, mouthrinses, gels or varnishes) for preventing dental caries in children and adolescents. Cochrane Database Syst Rev 2003; Issue 4. Art. No.: CD002782

6. Marinho VC, Higgins JP, Logan S, Sheiham A. Fluoride varnishes for preventing dental caries in children and adolescents. Cochrane Database Syst Rev 2002; Issue 1. Art. No.: CD002279

7. Marinho VC, Higgins JP, Logan S, Sheiham A. Fluoride gels for preventing dental caries in children and adolescents. Cochrane Database Syst Rev 2002; Issue 1. Art. No.: CD002280

8. Marinho VCC, Higgins JPT, Logan S, Sheiham A. Topical fluoride (toothpastes, mouthrinses, gels or varnishes) for preventing dental caries in children and adolescents. Cochrane Database of Syst Rev 2003, Issue 4. Art. No.: CD002782.

Evidence-Based Dentistry (2006) 7, 38-39.

doi:10.1038/sj.ebd.6400398 